\title{
Clima organizacional en Institutos de Investigaciones del Sector Salud Caso: Universidad del Zulia
}

\author{
Urdaneta Q., Omar R.* \\ Álvarez M., Carmen J.** \\ Urdaneta Q., Milagros del V.***
}

\section{Resumen}

El clima organizacional es un tema de gran interés para las organizaciones en la lucha por mejorar el ambiente de trabajo. El objetivo de este estudio fue determinar el clima organizacional en los Institutos de Investigaciones del Sector Salud de la Universidad del Zulia. La investigación fue descriptiva, aplicada, de campo y transeccional. Se consideraron como indicadores: los estilos de liderazgo, la comunicación y la planta física. Se empleó un cuestionario tipo Lickert aplicando la técnica del censo, con un nivel de confiabilidad del 0.96. Los resultados se analizaron a través de estadísticas descriptivas (frecuencias relativas y absolutas), evidenciándose en los institutos de investigación objeto de estudio la presencia de un estilo de liderazgo autocrático $(43,2 \%)$. La existencia de una comunicación en sentido descendente entre el personal que conforman las instituciones $(87,4 \%)$ y finalmente, una prevalencia en el descontento de los trabajadores con la planta física donde laboran $(60,8 \%)$. Se concluye que el clima organizacional detectado pod ría catalogarse como poco ideal para el óptimo desarrollo de los procesos que determinan la productividad del personal y, en definitiva, de todo el sistema organizacional.

Palabras clave: Clima organizacional, estilos de liderazgo, comunicación, planta física.

\section{Recibido: 09-01-08. Aceptado: 28-05-09}

* Economista, de la Universidad del Zulia (LUZ). Magíster en Gerencia Empresarial, Universidad Rafael Belloso Chacín (URBE). Autor para la correspondencia. E mail: omar_urdaneta_q@yahoo.com

** Doctora en Odontología. Profesora Asociada de la Universidad del Zulia. Instituto de Investigaciones de la Facultad de Odontología.E mail: carmitaalvarez@gmail.com

*** Doctora en Odontología. Profesora Titular de la Universidad del Zulia. División de Estudios para Graduados de la Facultad de Odontología. E mail: miliur69@hotmail.com 


\title{
Organizational Climate in Research Institutes for the Health Sector at the University of Zulia
}

\begin{abstract}
The organizational climate is a subject of great interest for organizations in the struggle to improve their work atmosphere. The objective of this study was to determine the organizational climate in Research Institutes for the Health Sector at the University of Zulia. The research was descriptive, applied, of the field type and trans-sectional. The following were considered as indicators: leadership styles, communication and the physical plant. A Lickert-type questionnaire was used, applying the census technique, with a reliability level of 0.96 . Results were analyzed using descriptive statistics (relative and absolute frequencies), demonstrating the presence of an autocratic leadership style $(43.2 \%)$ in the research institutes under study; the existence of downward communication among personnel that make up institutions ( $87.4 \%$ ); and finally, prevalence of the workers' displeasure with the physical plant where they labor $(60.8 \%)$. Conclusions are that the detected organizational climate could be catalogued as hardly ideal for optimal development of the processes that determine personnel productivity and, definitely, of the entire organizational system.
\end{abstract}

Key words: Organizational climate, leadership styles, communication, physical plant.

\section{Introducción}

En la actualidad el clima organizacional es un tema de gran importancia para las organizaciones con disposición a lograr un continuo mejoramiento del ambiente de trabajo, considerando a sus miembros como principal objeto de estudio.

El clima organizacional es el resultado de las percepciones que tengan los trabajadores en cuanto a los factores organizacionales existentes en el lugar de trabajo, tales como: los estilos de liderazgo adoptados por la gerencia, la estructura organizativa y las relaciones entre los empleados (Gonçalves, 1997).

En este sentido, estos elementos se suman para formar un ambiente laboral particular dotado de sus propias características, que representa en forma alguna, la personalidad de una organización e influye en el comportamiento de un individuo en su trabajo (Caligiore et al., 2003).

El objetivo de esta investigación fue determinar el clima organizacional en Institutos de Investigaciones del Sector Salud de la Universidad del Zulia, considerando como indicadores de estudio los estilos de liderazgo, comunicación y planta física.

Para la realización del trabajo se empleó una metodología tipo descriptiva, aplicada, de campo no experimental y transeccional.

La población estuvo conformada por cuatro (4) Institutos de Investigaciones (Clínicas, Biológicas, Medicina e Higiene del Trabajo y Odontológicas) de las Facultades de Medicina y Odontología de la Universidad del Zulia. Se aplicó un censo poblacional mediante la técnica de encuesta la cual fue aplicada a los investigadores, utilizando para ello un cuestionario tipo escala de Lickert, con las siguientes 
opciones de respuesta: Totalmente de Acuerdo (TA), Parcialmente de Acuerdo (PA), Ni de Acuerdo Ni en Desacuerdo (NAND), Parcialmente en Desacuerdo (PD) y Totalmente en Desacuerdo (TD).

El instrumento fue validado mediante el juicio de cinco (5) expertos del área de clima organizacional. También se aplicó una prueba piloto y se verificó la validez aplicando la prueba Alfa Cronbach, utilizando el Paquete Estadístico para las Ciencias Sociales (SPSS), versión 16.0 , con un $0,96 \%$ de confiabilidad.

Así mismo, el instrumento aplicado constó de 15 ítems, los cuales abordaron los indicadores en estudio de la siguiente manera: Estilos de liderazgo: ítem 1 (liberal), 2 y 4 (autocrático), y 3 (democrático); Comunicación: ítems 5 y 6 (descendente), 7 (ascendente), 8 (horizontal) y 9 (diagonal); planta física: ítems 10 al 15.

\section{Clima organizacional}

Arancibia (2007:1) señala que "el clima es una variable que media entre la estructura, procesos, metas y objetivos de la empresa, por un lado y las personas, sus actitudes, comportamiento y desempeño en el trabajo, por otro".

Para Castillo et al. (2000), un clima organizacional estable y flexible promueve en el personal logros en el largo plazo, por el contrario, una organización con una disciplina demasiado rígida, con demasiadas presiones al personal, sólo obtendrá logros a corto plazo.

Por su parte, Chiavenato (2000) considera que el clima organizacional está íntimamente relacionado con la motivación, al tener una motivación elevada el clima organizacional tiende a ser más alto proporcionando de éste modo satisfacción y mayor participación entre las personas. De forma inversa, un clima bajo proporciona estados de desinterés entre el personal, apatía, insatisfacción y hasta depresión en algunos casos. Por tanto, puede decirse que las propiedades motivacionales van a estar supeditadas por la forma en que los directivos manejen y controlen el ambiente interno de la empresa hacia los empleados.

Con base en lo antes expuesto, se puede decir que los estilos de liderazgo del jefe y la relación con el resto del personal, van a moldear el ambiente laboral. De allí que, Toro et al. (2004:3) expresan que "un buen clima promueve en cada uno de los miembros de la empresa, el buscar nuevas maneras de realizar su labor y ser más efectivos".

Por otra parte, un adecuado clima organizacional propicia el buen desempeño de la empresa, logrando ser factor de distinción e influencia en el comportamiento de los miembros que la conforman; es decir, es la expresión personal sobre la forma de ver los trabajadores y directivos a la organización a la que pertenecen. Así lo señala Ríos (2003: 1) cuando expresa que el clima organizacional "es la percepción individual que tiene cada uno de los integrantes acerca de las características y cualidades de su organización".

Así mismo, la forma de comportarse un individuo en el trabajo no depende solamente de sus características personales sino también de la forma en que éste percibe su clima de trabajo y los componentes de su organización (Sandoval, 2004). Por ello, Varela (1991) plantea que para la existencia de un buen cli- 
ma deben estar presentes factores físicos y psicológicos; entre los factores físicos está un lugar de trabajo confortable, donde los empleados puedan encontrar todas las herramientas para desarrollar de una manera eficiente su labor, el lugar debe ser iluminado, limpio y ubicado de acuerdo a sus necesidades.

Los aspectos psicológicos se refieren a las relaciones existentes entre el personal que conforma la organización, bien sea entre los empleados o entre los superiores y éstos en lo que respecta al trato, al incentivo y la forma en que los promueven por su eficiente labor. Si el individuo se siente motivado para efectuar sus tareas, bien sea por llevar buena comunicación con sus compañeros de trabajo o por su salario, puede tener resultados sorprendentes en el desarrollo de nuevas ideas en la resolución de problemas (Varela, 1991).

Algunas de las dimensiones o aspectos de análisis inherentes al clima organizacional, ampliamente identificados y definidos en la literatura, al caracterizar la dinámica de las organizaciones, se refieren a los estilos de liderazgo como imagen gerencial, la comunicación y el espacio físico.

\subsection{Liderazgo}

Según Hughes et al. (2007: 6)"el liderazgo es un fenómeno complejo que involucra al líder, a los seguidores y la situación". Es probable que con los líderes con disposición a la tranquilidad y sin ataques o menosprecio hacia los subordinados, se obtenga información completa y oportuna, a diferencia del caso de jefes con temperamentos explosivos.
Según Gibson et al. (2006), el liderazgo se produce cuando un individuo perteneciente a un grupo de personas modifica la motivación o las capacidades de los demás hacia un interés común que beneficie a todos por igual.

Para Bateman et al. (2005:366), "un líder es alguien que influye sobre otros para alcanzar metas, mientras más número de seguidores existan, mayor será la influencia".

Igualmente, Koontz et al. (2004: 532), definen el liderazgo como "el arte o proceso de influir en las personas para que se esfuercen voluntaria y entusiastamente en el cumplimiento de metas grupales".

Por su parte Chiavenato (2002: 557), considera el liderazgo como "un proceso clave en toda organización y ha sido una preocupación constante tanto de las organizaciones que lo necesitan como de los teóricos".

Lippitt et al. (1960). proponen tres estilos básicos de liderazgo: autocrático, liberal y democrático.

Liderazgo autocrático: la autoridad está centrada en el jefe y los subordinados no tienen ninguna libertad para tomar decisiones.

Liderazgo liberal: se da plena libertad de tomar decisiones al personal. El comportamiento del líder es evasivo y sin firmeza. Los grupos sometidos al liderazgo liberal no se desempeñan bien en cuanto a la calidad del trabajo, y presentan fuertes señales de individualismo, insatisfacción, agresividad y poco respeto hacia el líder.

Liderazgo democrático: el líder es comunicativo en extremo, estimula la participación de las personas y se preo- 
cupa igualmente por el trabajo y por el grupo. Actúa como facilitador para orientar al grupo, ayudándolos a la definición de los problemas y en las soluciones, coordinando actividades y sugiriendo ideas.

Cortés (2004), señala que los mayores niveles de motivación en el ambiente laboral concuerdan con la existencia de liderazgos de tendencia democrática, donde la participación, el respeto y el compromiso son parte del trato que se les brinda a los empleados.

Luego de analizar las diferentes definiciones de liderazgo por los autores señalados, se concibe el término como un proceso de influir que tiene un individuo sobre otros y el de apoyarlos para que trabajen con entusiasmo en el logro de objetivos comunes. El líder es aquella persona con la capacidad de tomar la iniciativa, gestionar, convocar, promover, incentivar, motivar y evaluar a un grupo o equipo.

\subsection{Comunicación}

Para Bateman et al. (2005:452), "la comunicación es la transmisión de información y significado de una parte a otra a través del uso de símbolos compartidos".

Según Robbins (2004:284), "la comunicación no sólo es una transferencia de significados entre un grupo de personas, comprende además la transferencia y comprensión de significados". En este proceso de la comunicación, intervienen dos sujetos quienes se deben a una intención que debe ser transmitida por el transmisor quien se dirige al receptor y el mensaje se codifica y se transmite por un medio o canal al receptor, que al traducirlo decodifica la información.
Por su parte Chiavenato (2002: 520), define la comunicación "como el puente que permite a ese algo pasar de una persona a otra o de una organización a otra".

Gibson et al. (2006) y Chiavenato (2002), coinciden que en toda organización existen cuatro direcciones de comunicación: ascendente, descendente, horizontal y diagonal, las cuales servirán para establecer el marco de trabajo dentro del cual la comunicación ocurre en una empresa.

La comunicación ascendente logra el buen desempeño laboral de los gerentes, en ellos se incluyen las sugerencias, las reuniones de trabajo y los procedimientos de queja o atracción. Frecuentemente los directivos participan en la elaboración, orientación y capacitación de los programas para instruir a los empleados para que puedan mantener un contacto directo con ellos, ofreciendo luego recompensas por las ideas creadas.

Sin embargo, Newstrom (2007) considera que la comunicación ascendente trae problemas en cuanto a retrasos, lentitud en la información hacia los niveles superiores, el personal indeciso duda si debe llevar un problema hacia arriba, porque al hacerlo significa admitir un fracaso y por otra parte está la distorsión, que no es más que la alteración deliberada de un mensaje para alcanzar los objetivos personales de quien emita la información, causando cualquier inconveniente para quien reciba el mensaje para su codificación y tomar una decisión.

En cuanto a la comunicación descendente, Gibson et al. (2006) y Chiavenato (2002) plantean que va desde los niveles altos hacia los niveles inferiores, 
en este renglón se incluyen los memorando, los procedimientos, los comunicados de políticas y las publicaciones de la compañía.

Se considera a este tipo de comunicación inadecuada y poco acertada debido a la ausencia de información relacionada al trabajo, que puede crear un estrés innecesario entre los miembros de las empresas. De igual manera lo plantean Bateman et al. (1999:551), al referir que la comunicación descendente trae problemas como mala interpretación que se le da a los mensajes enviados de arriba hacia abajo, y los denominan filtrados, "cuando los mensajes pasan de una persona a otra y se pierde alguna información".

Según Newstrom (2007), la comunicación descendente en algunos casos se sobrecarga de comunicación, en el sentido que los administradores le entregan a los empleados grandes volúmenes de información y éstos quedan abrumados ante la dificultad de procesar tal información. Las claves para una mejor comunicación son: sentido de oportunidad y su calidad, no su cantidad, siendo entregada en el momento que lo estime oportuno.

Chiavenato (2002), considera la comunicación horizontal necesaria para los diversos departamentos que conforman una organización, reforzando la coordinación y la integración de las diversas funciones en los procesos de producción. Por otra parte, para el mismo autor la comunicación diagonal se da en situaciones donde los departamentos de una organización no pueden comunicarse efectivamente a través de otros canales. La preferencia de un canal sobre el otro va a depender de la naturaleza e importancia, aunado al estilo de comportamiento entre los departamentos, considerando para ello si el mensaje es rutinario o no rutinario.

El primer tipo de mensaje tiende a ser directo y con un mínimo de ambigüedad mientras que los no rutinarios son complicados y tienden a confundir. Los gerentes pueden comunicar los mensajes rutinarios a través de los canales que no poseen mucha riqueza (panfletos, boletines, informes generales, memorandos y cartas), mientras que pueden comunicar los mensajes no rutinarios a través de los canales ricos como el correo electrónico, teléfono y conversaciones cara a cara.

Un gerente de alto rendimiento estará más sensible a la adecuada selección del canal a la hora de transmitir la información.

\subsection{Planta física}

Según Jorber et al. (2007:180), la planta física se trata del "entorno en el que se entrega el servicio y cualquier bien tangible que facilite el rendimiento y la comunicación".

Peter et al. (2006), argumentan que el ambiente físico incluye todos los aspectos tangibles divididos en espaciales (producto y marcas) y los no espaciales (temperatura, humedad ambiental, iluminación e intensidad del ruido).

En este sentido, Ivancevich (2005) expone que toda persona actúa en respuesta a las tendencias internas y elecciones, así como a las influencias ambientales. Es por ello la importancia de desarrollar en el empleado una actitud positiva respecto a su comportamiento en el trabajo y al entorno donde éste se maneja. 
Para Marchant (2006) resulta interesante observar cómo en la realidad de la organización, factores como el espacio físico, el entorno y la infraestructura con que cuentan las personas en su lugar de trabajo, resultan elementos importantes con influencia fuerte en su quehacer laboral.

Por su parte, Chiavenato (2002) plantea la importancia de una adaptación ambiental por parte de las organizaciones, es decir, la disposición de ambientes de trabajo acordes a la buena atención de las personas que visitan la empresa en busca de bienes.

Según la Organización Internacional del Trabajo en sus artículos 7 al 19, Convenio C 120 (1964), los locales utilizados por los trabajadores deben estar en buen estado de conservación en lo referente a iluminación, ventilación, muebles y equipos. No deben atentar la salud de los trabajadores, manteniendo en condiciones de salubridad las instalaciones sanitarias y protegidos contra vibraciones y olores tóxicos si los hubiere. Además, contar con un equipo de primeros auxilios en caso de eventualidades.
Atendiendo a estas consideraciones, el soporte físico se refiere a aspectos concretos de la edificación, la manera de cómo están dispuestas las oficinas y salas de atención al cliente, mobiliario, ubicación geográfica del negocio dentro de la ciudad, facilidad de transporte, entre otros.

De allí que, el ambiente físico conformado por elementos como la temperatura, el nivel de ruido, el tipo e intensidad de iluminación, pueden influir en el estado de ánimo de la persona que ejecuta su trabajo dentro de la empresa y de las personas que esperan ser atendidas, por ello la importancia de mantener en condiciones apropiadas la planta física, que repercutirá directa e indirectamente en la productividad de los trabajadores, en la satisfacción de los clientes y por ende en el logro de los objetivos de la empresa.

\section{Resultados}

En relación al liderazgo gerencial presente en los Institutos de Investigaciones del Sector Salud (Tabla 1), se identificaron varios estilos, prevaleciendo el li-

Tabla 1

Estilos de Liderazgo

\begin{tabular}{lcccccc}
\hline \multicolumn{1}{c}{ ítems } & TA \% & PA \% & NAND \% & PD \% & TD \% & Total \\
\hline $\begin{array}{l}\text { 1. El gerente le otorga plena libertad de tomar } \\
\text { decisiones al personal (Líder liberal). }\end{array}$ & 17,90 & 13,70 & 9,40 & 15,80 & 43,20 & 100,00 \\
$\begin{array}{l}\text { 2. En la institución se hace únicamente lo que } \\
\text { dice el jefe (Líder autocrático). }\end{array}$ & 65,20 & 0,00 & 0,00 & 0,00 & 34,80 & 100,00 \\
$\begin{array}{l}\text { 3. Los gerentes estimulan la participación de } \\
\text { los trabajadores (Líder democrático). }\end{array}$ & 42,10 & 17,90 & 2,10 & 6,30 & 31,60 & 100,00 \\
$\begin{array}{l}\text { 4. Los gerentes consideran poco importante } \\
\text { relacionarse con el personal (Líder autocrático) }\end{array}$ & 51,60 & 7,40 & 0,00 & 21,00 & 20,00 & 100,00 \\
\hline
\end{tabular}

Fuente: Elaboración propia. 
derazgo autocrático, dado que el $43,2 \%$ de la población estudiada estuvo en total desacuerdo con el ítem "El gerente le otorga plena libertad de tomar decisiones al personal", a diferencia de un $17,90 \%$ que estuvo totalmente de acuerdo.

Respecto a que si "en la institución se hace únicamente lo que dice el jefe", un $65,2 \%$ refirió estar totalmente de acuerdo, a diferencia de un $34,8 \%$ que estuvo en total desacuerdo. Igualmente, al consultar si "los gerentes estimulan la participación de los trabajadores", el $51,6 \%$ estuvo totalmente de acuerdo, a diferencia de un $20 \%$ que refirió estar totalmente en desacuerdo.

El estilo gerencial aquí identificado es denominado por Lippitt et al. (1960), como liderazgo autocrático; al respecto, Koontz et al. (2004:538), afirman que "el líder autocrático impone y espera cumplimiento, es dogmático y tiene la capacidad de negar u otorgar premios y castigos". Al mismo tiempo, Bateman et al. (1999), lla- man a este tipo de líder con Poder Legítimo, el cual posee la autoridad de decir a los demás qué hacer y los empleados están obligados a cumplir sus mandatos.

En cuanto al indicador Comunicación (Tabla 2), destaca que un $87,4 \%$ estuvo totalmente de acuerdo en que "se debe acatar la orden y ejecutarla", a diferencia de un $4,2 \%$ que respondió estar en total desacuerdo con este planteamiento.

Por otra parte, un $55,9 \%$ respondió estar totalmente de acuerdo y aceptar que "se comunican las políticas y controles, sin permitir la participación del personal", a diferencia de un $31,5 \%$ que estuvo totalmente en desacuerdo.

Para Bateman et al. (1999), este tipo de situaciones obedece a comunicaciones descendentes, donde las personas deben recibir la información que necesitan para realizar sus tareas.

Según Gibson et al. (2001), una comunicación hacia abajo frecuentemente es inadecuada y poco acertada. Por ello,

\section{Tabla 2}

Comunicación

\begin{tabular}{|c|c|c|c|c|c|c|}
\hline Ítems & TA \% & PA\% & NAND $\%$ & PD \% & TD \% & Total \\
\hline $\begin{array}{l}\text { 5. Se debe acatar la orden y ejecutarla } \\
\text { (Descendente). }\end{array}$ & 87,40 & 0,00 & 4,20 & 4,20 & 4,20 & 100,00 \\
\hline $\begin{array}{l}\text { 6. Se comunican las políticas, controles sin } \\
\text { permitir la participación del perosnal }\end{array}$ & 55,90 & 0,00 & 0,00 & 12,60 & 31,50 & 100,00 \\
\hline
\end{tabular}
(Descendente).

7. Se puede dar sugerencias a los jefes inmediatos (Ascendente).

8. Existe comunciación entre los departamentos que conforman la institución (Horizontal).

9. Cuesta obtener información de otro departamento cuando se necesita (Diagonal).

$\begin{array}{llllll}32,60 & 0,00 & 0,00 & 9,50 & 57,90 & 100,00 \\ 34,70 & 0,00 & 0,00 & 0,00 & 65,30 & 100,00\end{array}$

Fuente: Elaboración propia. 
son necesarios los cuadros de sugerencias, las reuniones de trabajo y los procedimientos de queja, que ayudan a fluir ese proceso de comunicación hacia arriba, haciendo efectivo el logro de las metas de la institución. Por su parte, Chiavenato (2004) sugiere la incorporación de programas de sugerencias ofrecido a los empleados y considera tomar en cuenta a aquellos trabajadores que suministren ideas que tengan aplicación y generen buenos resultados para la organización.

El estudio reportó que cada departamento evalúa sus problemas aisladamente, dado que al indagar si existe una comunicación entre los departamentos que conforman la institución, un 65,3\% estuvo en total desacuerdo, a diferencia de un $34,7 \%$ que respondió estar totalmente de acuerdo. Bateman et al. (1999), recomiendan para este tipo de situaciones una comunicación horizontal, donde se pueda intercambiar información entre los departamentos, brindando en ese sentido apoyo social y emocional a los trabajadores.

Finalmente, en el indicador Planta Física (Tabla 3), un 60,8\% estuvo en total desacuerdo con respecto a que "la estructura física de la institución donde labora se encuentra en perfectas condiciones". Sin embargo, un $21 \%$ respondió estar totalmente de acuerdo.

$\mathrm{Al}$ considerar si "el área donde se ejercen las labores es cómoda y espaciosa", un $55,8 \%$ respondió estar totalmente en desacuerdo, pero un $42,1 \%$ estuvo totalmente de acuerdo. Estos resultados coinciden con lo reportado en el estudio de Villa et al. (2005), donde refieren que el clima organizacional presenta condiciones no favorables en el entorno físico donde se labora, situando el factor comodidad en un nivel bajo.

De igual manera, al consultar la disposición de tecnología que cubra todas las necesidades del personal en la institución, un 55,8\% respondió estar totalmen-

Tabla 3

Planta Física

\begin{tabular}{lcccccc}
\hline \multicolumn{1}{c}{ Ítems } & TA \% & PA \% & NAND \% & PD \% & TD \% & TOTAL \\
\hline $\begin{array}{l}\text { 10. La estructura física de la institución se } \\
\text { encuentra en perfectas condiciones. }\end{array}$ & 21,00 & 3,60 & 0,00 & 14,60 & 60,80 & 100,00 \\
$\begin{array}{l}\text { 11. En el área donde trabajo es incomoda para } \\
\text { ejecutar mis labores cotidianas. }\end{array}$ & 39,00 & 12,60 & 3,20 & 14,70 & 30,50 & 100,00 \\
$\begin{array}{l}\text { 12. El área donde ejerzo mis labores es } \\
\text { cómoda y espaciosa. }\end{array}$ & 42,10 & 0,00 & 2,10 & 0,00 & 55,80 & 100,00 \\
$\begin{array}{l}\text { 13. Los muebles del área de trabajo no están } \\
\text { acorde a los requerimientos de mis funciones. }\end{array}$ & 50,50 & 0,00 & 0,00 & 22,10 & 27,40 & 100,00 \\
$\begin{array}{l}\text { 14. Dispongo de una tecnología que cubre } \\
\text { todas las necesidades de la institución. }\end{array}$ & 32,60 & 11,60 & 0,00 & 0,00 & 55,80 & 100,00 \\
$\begin{array}{l}\text { 15. Los equipos tecnológicos no se adecuan a } \\
\text { las necesidades de la institución. }\end{array}$ & 56,80 & 0,00 & 0,00 & 5,30 & 37,90 & 100,00 \\
\hline
\end{tabular}

Fuente: Elaboración propia. 
te en desacuerdo, a diferencia de un $32,6 \%$ que estuvo totalmente de acuerdo. En este sentido, se considera la tecnología de punta de vital importancia para enfrentar la demanda creciente con la globalización de los mercados. Al respecto, Marchant (2003) señala que mientras mejor es la percepción de los miembros de la organización en lo atinente a las condiciones ambientales físicas del trabajo, tales como estructura, distribución y acondicionamiento de los espacios físicos del trabajo, mejor es también su desempeño laboral.

Contrario a lo aquí reportado, Marchant (2003) observó en su estudio que los funcionarios manejaban apropiadamente los equipos y/o maquinarias, practicaban las normas de seguridad del trabajo, cumplían mejor con sus horarios, se comunicaban fluidamente con sus compañeros y jefaturas, intercambiaban información y opiniones entre ellos, y aportaban ideas y acciones concretas para enfrentar situaciones problemáticas. Condiciones éstas que hacen del ambiente de trabajo un escenario armónico e integral.

\section{Conclusiones}

Los resultados obtenidos indican que en los institutos de investigaciones del Sector Salud de La Universidad del Zulia existe un estilo de líder autócrata, el cual impone las normas y espera sean cumplidas. Esto afecta las relaciones entre directivos y personal, en el sentido de la ausencia de valorización de las ideas del personal por parte de los jefes. Cuando las personas tienen una motivación se comprometen con su trabajo por el reto que supone y porque disfrutan con ello.

Es fundamental que a los empleados se les estimule haciéndolos sentir como una parte fundamental de la empresa, comprometiéndolos con ella no sólo con incentivos de tipo económico, sino con reconocimientos de tipo personal. El ánimo que se recibe de los directivos realmente fomenta la creatividad en el cargo, pero ésta se mejora verdaderamente cuando el empleado percibe que toda la organización lo respalda.

El gerente de hoy debe ser una mezcla de líder autocrático, liberal y democrático en el momento que lo requiera, así como también conocer todos aquellos aspectos que pueden afectar una organización, estar preparado para enfrentar las adversidades, y junto con el equipo humano que dirige contrarrestarlas, y aprender de ellas para experiencias futuras.

En torno a la dirección de la comunicación en los institutos de investigaciones del área salud, la más empleada es la descendente, donde las normas y procedimientos son canalizados por la dirección y enviados a los trabajadores. En tal sentido, se usa esta dirección de la comunicación para informar, dirigir, coordinar y evaluar a los subordinados. En un esfuerzo por comprender y argumentar en pro y contra del tipo de comunicación presente, puede señalarse que las personas que toman las decisiones necesitan estos métodos para dictar a sus subordinados las pautas a seguir, qué políticas adoptar pero también dar conocimiento a los empleados sobre la organización y retroalimentación de sus fuerzas. 
La planta física de las instituciones estudiadas se encuentra en condiciones inadecuadas para su gestión. Esto es lamentable porque la infraestructura, así como los materiales usados para su construcción, son piezas claves y muchas veces determinantes, para su competitividad en el campo científico. El papel de la tecnología debe verse como una herramienta que facilite el trabajo y establezca facilidades y ventajas en el quehacer diario de los institutos de investigaciones del área salud.

Finalmente, se considera que el clima organizacional detectado a través de los indicadores seleccionados, podría catalogarse como poco ideal para el óptimo desarrollo de los procesos que determinan la productividad del personal y, en definitiva, de todo el sistema organizacional.

\section{Referencias bibliográficas}

Arancibia, Joselyn (2007). Clima como Herramienta de Gestión. Disponible en: http://www.eumed.net/libros/2007a/ 223/jam.htm. (Consulta: 2007, Enero 24)

Bateman, Thomas y Snell, Scott (1999) Administración. Una Ventaja Competitiva. Editorial: McGraw Hill. Primera Edición. México.

Bateman, Thomas. y Snell, Scott. (2005). Administración. Un Nuevo Panorama Competitivo. Sexta Edición. Editorial: McGraw-Hill. México.

Caligiore, Irene y Díaz, Juan (2003). Clima Organizacional y Desempeño de los Docentes en la ULA: Estudio de un caso. Disponible en: http://www.serbi.luz.edu.ve/scielo.php?script=sci_ arttext\&pid=S1315-99842003012000 008\&lng=es\&nrm=is. (Consulta: 2007, Enero 14)
Castillo, Carola; Del Pino, Nicole; Espinoza, Vita (2000). Identidad Corporativa. Disponible en: http://www.rrppnet. com.ar/culturaorganizacional.htm. (Consulta: 2007, Enero 22).

Cortés Mejía, Alejandra (2004). Estilos de liderazgo y motivación laboral en el ambiente educativo. Rev. Ciencias Sociales. Volumen IV No 106 , pp 203214. Disponible en: http://redalyc. uaemex.mx/redalyc/pdf/153/ 153106 15.pdf. (Consulta: 2007, Agosto 8).

Chiavenato, Idalberto (2000). Administración de Recursos Humanos. Editorial: Mc. Graw Hill. Quinta Edición. Bogotá.

Chiavenato, Idalberto (2002). Administración en los Nuevos Tiempos. Editorial: Mc. Graw Hill. Bogota.

Chiavenato, Idalberto (2004). Gestión del TaIento Humano. Editorial: Mc. Graw Hill. Cuarta Edición. Bogotá.

Gibson, James; Ivancevich, Johns y Donnely, James. (2001). Las Organizaciones. Editorial: Mc Graw Hill. Octava edición. México.

Gibson, James; Ivancevich, Johns; Donnelly, James y Konopaske, Robert. (2006). Organizaciones. Comportamiento, estructura, procesos. Duodésima edición. Editorial: McGraw Hill. México.

Gonçalves, Alexis (1997). Dimensiones del Clima Organizacional. Disponible en: http://www.calidad.org/articles/ dec97/2dec97.htm. (Consulta:2007, Enero 16).

Hughes, Richard; Ginnett, Robert y Curphy, Gordon (2007). Liderazgo. India. Quinta edición. Mc Graw-Hill.

Ivancevich, Johns. (2005). Administración de Recursos Humanos. Novena edición. Editorial: Mc. Graw Hill. México.

Jorber, David y Fahy, John ((2007). Fundamentos del Marketing. Segunda edición. Editorial: Mc. Graw Hill. España. 
Koontz, Harold y Weinrich, Heinz (2004). Administración. Una perspectiva global. Editorial: Mc Graw Hill. Segunda edición. México.

Lippitt, Ronald y White, Ralph (1960). Autocracy and Democracy: An Experimental Inquiry. Nueva York, Harper and Row.

Marchant R, Loreto (2003). Estudio descriptivo de la influencia del clima organizacional sobre las personas y su trabajo en empresas medianas de la Quinta Región. Tesis de magíster U. de Valparaíso, Chile.

Marchant R, Loreto. (2006). Factores organizacionales críticos para fortalecer el alineamiento estratégico del personal. Ciencias Sociales Online, Marzo 2006, Vol. III, No. 1. Universidad de Viña del Mar - Chile pp 58-69. Disponible en: http://www.uvm.cl/csonline/ 2006 1/pdf/factoresorganizacionales.pdf. (Consulta: 2007, Septiembre 21).

Newstrom, John (2007). Comportamiento Humano en el Trabajo. Duodécima edición. Editorial: Mc. Graw Hill. México.

Organización Internacional del Trabajo (1964). C120 Convenio sobre la higiene (comercio y oficinas). Disponible en: http://www.ilo.org/ilolex/cgi-lex/conv ds.pl?C120. (Consulta: 2007, Enero 22).

Peter, Paul y Olson, Jerry ((2006). Comportamiento del Consumidor y Estrategia de Marketing. Séptima edición. Editorial: Mc. Graw Hill. México.
Ríos, Amílcar (2003). Cultura Organizacional. Disponible en. http://www.geocities.com/amirhali/-fpclass/cultura-organizacional.htm. (Consulta: 2006, Agosto 30).

Robbins, Stephen (2004). Comportamiento Organizacional. México. Editorial: Prentice May.

Sandoval Caraveo, María del Carmen (2004), Concepto y Dimensiones del Clima Organizacional, Hitos de Ciencias Económico Administrativas, Año 10, № 27, México, Universidad Juárez Autónoma de Tabasco, pp 83-88.Disponible en http://www.ujat.mx/publicaciones/hitos/ediciones/27/index. html\#. (Consulta: 2007, Marzo 06)

Toro, Natalia; Ochoa, Luz; Vargas, Adrián (2004). Clima Organizacional: Promotor de la Creatividad en la Empresa. Disponible en: http://72.14. 209.104/search?q=cache:-FG1C7 MxuScJ:www.manizales.unal.edu.co /modules/unrev_creando/documentos/Clima.pdf+clima+organizacional $\& \mathrm{hl}=\mathrm{es} \& \mathrm{gl}=\mathrm{ve} \& \mathrm{ct}=\mathrm{clnk} \& \mathrm{~cd}=20$. (Consulta: 2007, Enero 16)

Varela, Rodrigo (1991). Innovación Empresarial: Un Nuevo Enfoque de Desarrollo. ICESI. Cali - Valle.

Villa, Eulalia; Pons, Ramón y Castellano, José (2005). Clima Organizacional y Control de Gestión en la Educación Superior. El caso de una Universidad. Disponible en: http://www.dict. uh.cu/Revistas/Educ_Sup/032005 /Art09.pdf. (Consulta: 2007, Septiembre 22). 